\title{
COMPACT COMPOSITION OPERATORS ON SPACES OF BOUNDARY-REGULAR HOLOMORPHIC FUNCTIONS
}

\author{
JOEL H. SHAPIRO
}

\begin{abstract}
We consider holomorphic functions $\phi$ taking the unit disc $U$ into itself, and Banach spaces $X$ consisting of functions holomorphic in $U$ and continuous on its closure; and show that under some natural hypotheses on $X$ : if $\phi$ induces a compact composition operator on $X$, then $\phi(U)$ must be a relatively compact subset of $U$. Spaces $X$ which satisfy the hypotheses of this theorem include the disc algebra, "heavily" weighted Dirichlet spaces, spaces of holomorphic Lipschitz functions, and the space of functions with derivative in a Hardy space $H^{p}(p \geq 1)$. It is well known that the theorem is not true for "large" spaces such as the Hardy and Bergman spaces. Surprisingly, it also fails in "very small spaces," such as the Hilbert space of holomorphic functions $f(z)=\sum a_{n} z^{n}$ determined by the condition $\sum\left|a_{n}\right|^{2} \exp (\sqrt{n})<\infty$. The property of Möbiusinvariance plays a crucial and mysterious role in these matters.
\end{abstract}

1. Introduction. Throughout this paper $\phi$ denotes a nonconstant holomorphic function on the open unit disc $U$ of the complex plane, with $\phi(U) \subset U$. Thus $\phi$ induces on $H(U)$, the space of functions holomorphic on $U$, a composition operator $C_{\phi}$ defined by the equation $C_{\phi} f=f \circ \phi(f \in H(U))$. There has been growing interest in the study of composition operators on Banach spaces of functions holomorphic on $U$, the idea being to connect the behavior of the operator $C_{\phi}$ with the function theoretic properties of $\phi$. The most popular setting for this research has been the Hardy space $H^{p}$, but more recently the weighted Bergman and Dirichlet spaces have begun to assert themselves (see $[\mathbf{7}, \mathbf{1 5}]$, for example). Recent work on composition operators in these spaces includes studies of spectra [4], algebras generated by composition operators $[\mathbf{3}, \mathbf{9}]$, and compactness of composition operators $[\mathbf{7}, \mathbf{1 4}, \mathbf{1 5}]$.

In [7] the study of compact composition operators on various Bergman and Dirichlet spaces led to phenomena which, in addition to their intrinsic interest, also shed considerable light on the Hardy space situation. Here we carry the study of compactness a step further, into the realm of "small" Banach spaces $X$ consisting of holomorphic functions in the unit disc which extend continuously to the boundary, have norm stronger than the sup norm, and contain the polynomials.

In $\S 2$ we prove (Theorem 2.1) that: If such a space $X$ is invariant under composition with all conformal automorphisms of $U$, and if $C_{\phi}$ acts compactly on $X$, then $\phi(U)$ must be a relatively compact subset of $U$.

Received by the editors September 6, 1985 and, in revised form, February 7, 1986 and March $5,1986$.

1980 Mathematics Subject Classification (1985 Revision). Primary 46E15; Secondary 46B05, 30D05.

Key words and phrases. Compact operator, composition operator, disc algebra.

The author's research was supported in part by the National Science Foundation. 
Examples of spaces to which this result applied include:

(1) The disc algebra $A$, consisting of all $f \in H(U)$ which extend continuously to the closed unit disc.

(2) The heavily weighted Dirichlet spaces $D_{\alpha}$ consisting of all $f \in H(U)$ whose complex derivative $f^{\prime}$ is square integrable over $U$ with respect to the measure $\left(1-|z|^{2}\right)^{\alpha} d x d y$ for $-1<\alpha<0$.

(3) The Lipschitz spaces $\Lambda_{\alpha}, 0<\alpha \leq 1$, consisting of all $f \in H(U)$ which obey a Lipschitz condition with exponent $\alpha$.

(4) The spaces $S^{p}, p \geq 1$, consisting of all $f \in H(U)$ for which $f^{\prime} \in H^{p}$.

(5) The "integrated space" $X_{1}$ consisting of all $f \in X$ for which $f^{\prime} \in X$, where $X$ satisfies our hypotheses, and is preserved by multiplicatin by functions holomorphic in a neighborhood of the closed unit disc.

Special cases of this theorem have appeared before, the first instance having been observed by H. J. Schwartz [13] for the disc algebra (and also for $H^{\infty}$, the space of bounded analytic functions on $U$ ). His proof is worth noting, since it is so simple, and since it does not generalize in any obvious way to the other spaces in our list. If $C_{\phi}$ is compact on $A$, then a straightforward argument shows that it must take any sequence in $A$ that is convergent pointwise on $U$ to zero into a sequence that is norm convergent to zero. In particular the monomials $\left(z^{n}: n \geq 0\right)$ form such a sequence, for which the image sequence is $\left(\phi^{n}: n \geq 0\right)$. Thus $\left\|\phi^{n}\right\|_{\infty} \rightarrow 0$, so $\|\phi\|_{\infty}<1$.

The weighted Dirichlet spaces $D_{\alpha}$ are studied in [7] where, although it is the range $\alpha \geq 0$ that is of primary interest, the main results are proved for all $\alpha>$ -1. In particular, a Carleson measure characterization of compact composition operators is obtained there (Proposition 5.1), but it is not mentioned, nor is it at all obvious, that when $\alpha<0$ this characterization is equivalent to the requirement $\|\phi\|_{\infty}<1$. For purposes of orientation, the reader should notice that $D_{\alpha}$ is the classical Dirichlet space when $\alpha=0$, the Hardy space $H^{2}$ when $\alpha=1$, and a weighted Bergman space when $\alpha>1$. We wish to emphasize that when $\alpha \geq 0$, the case we are not considering here, $D_{\alpha}$ contains unbounded functions, and does support compact composition operataors $C_{\phi}$ for which $\|\phi\|_{\infty}=1$. Indeed, the study of such operators is the main object of the papers [7, 14 and 15].

Rather complicated characterizations of boundedness and compactness for composition operators on $S^{p}$ and $\Lambda_{\alpha}$ were obtained by Roan $[\mathbf{1 1}, \mathbf{1 2}]$. However, as MacCluer [8] has recently observed, there are errors in [11] leading to examples of maps $\phi$ which purport to induce compact composition operators on $S^{p}$, but which in fact do not. In the same paper MacCluer uses the device of Carleson measures to prove the $S^{p}$ case of Theorem 2.1, thus clarifying and correcting Roan's results on compactness.

The proof of Theorem 2.1 occupies the next section. An interesting feature is the role played by the apparently heavy-handed assumption that $X$ be Möbiusinvariant: one is tempted to try to replace it by the more natural requirement of rotation-invariance. Further motivation for such an improvement comes from a natural example: The space of power series absolutely convergent in the unit disc is rotation-invariant, but not Möbious-invariant $[\mathbf{1}, \mathbf{5}]$. Schwartz's original proof works here also, so the conclusion of Theorem 2.1 continues to hold in this setting. 
We will see in $\S 3$, however, that mere rotation-invariance does not guarantee the conclusion of Theorem 2.1: If enough boundary smoothness is required of the functions in $X$, then there will exist compact composition operators $C_{\phi}$ on $X$ with $\|\phi\|_{\infty}=1$.

Thus, the situation can be summed up roughly as follows. If $X$ is too large, in that it admits unbounded functions, or too small, in that too much smoothness is required of its boundary functions, then $C_{\phi}$ may be compact on $X$ even though $\|\phi\|_{\infty}=1$. However this cannot happen for spaces whose boundary functions have smoothness moderated in some mysterious fashion by the requirement of Möbiusinvariance.

I would like to thank Carl Cowen for showing me an example that led to Theorem 3.4, and to thank Roy Erickson and Wade Ramey for helpful conversations regarding this theorem. In particular, Ramey first suggested that a result of this sort might be true, and Erickson pointed out Hoeffding's inequality which plays a crucial role in its proof. I would also like to thank Lech Drewnowski for his insightful observations regarding generalizations of Theorem 2.1.

2. Compactness implies $\|\phi\|_{\infty}<1$. In this section $X$ will denote a Banach space of functions holomorphic in $U$ which satisfies the following axioms.

(X1) Boundary regularity: every function in $X$ extends continuously to the closed unit disc.

(X2) Naturality of norm: Every convergent sequence in $X$ must converge to the same limit function uniformly on compact subsets of $U$.

(X3) Nontriviality: $X$ contains the holomorphic polynomials.

(X4) Möbius-invariance: if $\gamma$ is a conformal automorphism of $U$ and $f \in X$, then $f \circ \gamma \in X$.

It follows from (X1), (X2), and the Closed Graph Theorem that the topology of $X$ is stronger than the sup norm topology. Similarly, any composition operator taking $X$ into itself must actually be continuous on $X$. In particular, by (X4) the conformal automorphisms of $U$ induce (topological) isomorphisms of $X$. We can now state our main result.

2.1. THEOREM. If $\phi$ induces a compact composition operator on $X$, then $\|\phi\|_{\infty}<1$.

Before proceeding with the proof, let us observe that axiom (X3) guarantees that the identity function $u(u(z) \equiv z)$ belongs to $X$, hence the same is true of $\phi=C_{\phi} u$, and more generally of its $n$th iterate $\phi_{n}=\phi \circ \phi \circ \cdots \circ \phi$ ( $n$ times). In particular, $\phi$ and all its iterates are continuous functions on the closed unit disc, and can therefore be evaluated without apology on the boundary. Incidentally, this is the only essential use made of axiom (X3).

It will be convenient to isolate the main body of the proof as a lemma.

2.2. Lemma. Suppose $\phi(0)=0$ and $C_{\phi}$ is compact on $X$. Then $\left\|\phi_{n}\right\|_{\infty} \rightarrow 0$.

PROOF. Let $X_{0}$ denote the subspace of $X$ consisting of functions which vanish at the origin. Axiom (X2) implies that $X_{0}$ is a closed subspace of $X$. It is clearly invariant under $C_{\phi}$, and is nontrivial since it contains $u$ as well as $\phi$ and all its iterates. Our proof depends on showing that the restriction $T$ of $C_{\phi}$ to $X_{0}$ has spectral radius $\rho(T)<1$. 
To this end, suppose $\lambda$ is a nonzero spectral point of $T$. Being the restriction of a compact operator to an invariant subspace, $T$ is also compact, hence $\lambda$ is an eigenvalue. Fix $f \in X_{0}$, an eigenfunction of $T$ for the eigenvalue $\lambda$. Thus $T f=\lambda f$, and there is a point $a \in U$ for which $f(a) \neq 0$. Let $\Delta$ denote the open disc of radius $(1+|a|) / 2$, centered at the origin. Now $\phi$ is not a rotation (if it were, then $C_{\phi}$ would be an isomorphism, contradicting its compactness), so the Schwarz Lemma asserts that $\phi(\Delta)$ is a relatively compact subset of $\Delta$. A second application of the Schwarz Lemma, this time to the (suitably normalized) restriction of $\phi$ to $\Delta$, shows that $\phi_{n}(a) \rightarrow 0$. Thus

$$
\lambda^{n} f(a)=T^{n} f(a)=f\left(\phi_{n}(a)\right) \rightarrow f(0)=0
$$

as $n \rightarrow \infty$. Since $f(a) \neq 0$ we must therefore have $|\lambda|<1$. The compactness of $T$ also forces its spectrum to consist of the point 0 along with an at most countable set of eigenvalues which can cluster only at 0 . Thus the spectral radius of $T$ is the magnitude of the largest eigenvalue of $T$, which we have just seen to be $<1$. The spectral radius formula now shows that

$$
\lim \left\|T^{n}\right\|^{1 / n}=\rho(T)<1 \quad(n \rightarrow \infty),
$$

so in particular $\left\|T^{n}\right\| \rightarrow 0$.

Let \|\|$_{X}$ denote the norm of $X$, and recall that $\phi_{n}=T^{n} u \in X$. Thus,

$$
\left\|\phi_{n}\right\|_{X}=\left\|T^{n} u\right\|_{X} \leq\left\|T^{n}\right\|\|u\|_{X} \rightarrow 0 \quad(n \rightarrow \infty) .
$$

As we remarked at the beginning of this section, the topology of $X$ is stronger than the one induced by the sup norm, hence $\left\|\phi_{n}\right\|_{\infty} \rightarrow 0$.

2.3. PROOF OF THEOREM 2.1. Suppose initially that $C_{\phi}$ is only assumed to be a bounded operator on $X$, and that $\phi(0)=0$. If $\|\phi\|_{\infty}=1$, then there is a rotation $\rho$ such that $\psi=\rho \circ \phi$ has a fixed point on the unit circle. Thus $\left\|\psi_{n}\right\|_{\infty}=1$ for all $n$, so by Lemma 2.2 the operator $C_{\psi}$ cannot be compact.

Since $C_{\psi}=C_{\phi} C_{\rho}$, where $C_{\rho}$ is an isomorphism of $X$, it follows that $C_{\phi}$ is not compact either. What we have just proved can be restated as follows: if $\phi(0)=0$ and $C_{\phi}$ is compact on $X$, then $\|\phi\|_{\infty}<1$.

Now suppose that $\phi(0)=a \neq 0$. Let $\gamma$ be the conformal automorphism of $U$ taking $a$ to 0 , and set $\psi=\gamma \circ \phi$. Recall that $C_{\gamma}$ is a bounded operator on $X$ as a consequence of axioms (X4) (used for the first time) and (X2). Thus $C_{\psi}=C_{\phi} C_{\gamma}$ is compact on $X$, so by the result of the last paragraph, $\|\psi\|_{\infty}<1$. Thus $\|\phi\|_{\infty}<1$, and the proof is complete.

2.4. REMARKS. (a) As mentioned in the Introduction, we will see in the next section that Theorem 2.1 does not hold if the automorphism group is replaced in axiom (X4) by the group of rotations.

(b) In all the examples listed in $\S 1$ (and in the Hardy and Bergman spaces as well), the converse of Theorem 2.1 holds in the following form: if $\phi \in X$ and $\|\phi\|_{\infty}<1$, then $C_{\phi}$ is compact on $X$. It would be of interest to know if this result holds for all spaces $X$ satisfying axioms (X1)-(X4). In $\S 4$ we will see that there are rotation-invariant spaces satisfying $(\mathrm{X} 1)-(\mathrm{X} 3)$ for which this is not true.

(c) As we mentioned earlier, the nontriviality axiom (X3) is used only in the weaker form: " $X$ contains the identity function on $U$." Lech Drewnowski has pointed out that, upon properly reorganizing the proof of Theorem 2.1, it is enough to assume only that $X$ contains a nonconstant function. Drewnowski also observes 
that under the original axioms (X1)-(X4), the functions in $X$ need not be assumed to be holomorphic (as long as $\phi$ is holomorphic).

(d) We do not know if the boundary continuity axiom (X1) can be replaced by the requirement that each $f \in X$ be merely bounded on $U$.

3. Compactness does not imply $\|\phi\|_{\infty}<1$. In this section we show that hypothesis (X4), the Möbius-invariance required of the spaces $X$ of the last section, cannot be weakened to "rotation-invariance." The main result, Theorem 3.4, yields a large class of rotation-invariant spaces which satisfy hypotheses (X1)-(X3), yet which support compact composition operators $C_{\phi}$ with $\|\phi\|_{\infty}=1$. These are "extremely small" spaces composed of functions holomorphic in $U$ with exceptionally high boundary regularity. They are constructed by using a coefficient multiplier to modify the elements of an initial space of "moderate size."

3.1. Notation. If $n$ is a positive integer, and $f \in H(U)$, then we write $\hat{f}(n)$ for the $n$th Taylor coefficient of $f$ in its expansion about the origin, and define the $n$th coefficient functional $\lambda_{n}$ on $H(U)$ by $\lambda_{n}(f)=\hat{f}(n)$.

3.2. Initial spaces. In what follows, $Y$ will always denote a Banach space of functions holomorphic on $U$, with norm \|\| , satisfying the following hypotheses.

(Y1) $Y$ contains all the holomorphic polynomials.

(Y2) $Y$ is rotation-invariant: if $f \in Y$ and $\rho$ is a rotation of $U(\rho(z)=\omega z$ for some complex number $\omega$ of modulus 1 ), then $f \circ \rho \in Y$.

(Y3) There exists a constant $M<\infty$ such that $|\hat{f}(n)| \leq M\|f\|$ for every $f \in Y$ and every nonnegative integer $n$.

(Y4) The sequence of monomials $\left(z^{n}: n \geq 0\right)$ is bounded in $Y$.

Such a space $Y$ will be called an initial space. Typical examples are, for $1 \leq$ $p \leq \infty$, the Hardy space $H^{p}$, and the space of functions $f \in H(U)$ with Taylor coefficient sequence $(\hat{f}(n): n \geq 0)$ in $l^{p}$.

3.3. Final spaces. Here $w=(w(n): n \geq 0)$ denotes an increasing sequence of positive numbers. For $f \in H(U)$, let $f_{w}$ be the formal power series $\sum w(n) \hat{f}(n) z^{n}$. For each initial space $Y$ we define $Y_{w}$ to be the collection of functions $f \in H(U)$ for which $f_{w}$ represents a member of $Y$. The natural norm \|\|$_{w}$, defined for $f \in Y_{w}$ by $\|f\|_{w}=\left\|f_{w}\right\|$, makes $Y_{w}$ into a Banach space isometrically isomorphic to $Y$.

It is easy to check that every such "final space" $Y_{w}$ is rotation invariant and satisfies axioms (X2) and (X3) of §2. The "naturality" axiom (X2) follows from the corresponding property of $Y$, which in turn follows from (Y3) and a normal families argument. As for (X3), it is clear that since $Y$ contains all the holomorphic polynomials, so does $Y_{w}$. We can now state the main result of this section, the first part of which has just been proved. In what follows, $[t]$ denotes the integer part of the real number $t$.

3.4. THEOREM. For any initial space $Y$, the final space $Y_{w}$ is rotationinvariant and satisfies axioms (X2) and (X3) of §2. If, in addition, there exists $0<\alpha<1$ such that

$$
\sum_{n=0}^{\infty} w([\alpha n]) / w(n)<\infty,
$$

then $Y_{w}$ also satisfies axiom (X1), but supports a compact composition operator $C_{\phi}$ with $\|\phi\|_{\infty}=1$. 
3.5. EXAMPLES. Take $Y=H^{2}$, so $Y_{w}$ is just $H^{2}(w)$, the space of functions $f$ holomorphic on $U$ for which $\|f\|_{w}=\left\{\sum|\hat{f}(n) w(n)|^{2}\right\}^{1 / 2}<\infty$.

(a) Fix $R>1$ and set $w(n)=R^{n}$. Then (3.4.1) is satisfied. This example is the prototype for Theorem 3.4, in fact it is easy to see directly that the function $\phi(z)=(1+z) / 2$, for which $\|\phi\|_{\infty}=1$, induces a compact composition operator on $H^{2}(w) . H^{2}(w)$ is just $H^{2}(R U)$, the Hardy space of the disc of radius $R$, and $\phi(R U)$ is a relatively compact subset of $R U$; so the result follows by transferring to $R U$ the well-known fact that any map taking the unit disc into a relatively compact subset of itself induces a compact composition operator on $H^{2}(U)$ (see, for example, $[\mathbf{1 4}$, Theorem 3.1]). This example, and the above proof, were shown to me by Carl Cowen.

(b) Let $w(n)=\exp \left(n^{a}\right)$ for some $0<a<1$. Then (3.4.1) is satisfied, but in contrast with the last example, each space $H^{2}(w)$ contains functions not continuable across any point of the unit circle. Note that if $a \geq \frac{1}{2}$, then, by a result of Carleson [2], $H^{2}(w)$ is a quasi-analytic class on the closed unit disc.

(c) Some nonexamples. Let $w(n)=n^{a}$ for $a>1$. Then (3.4.1) is not satisfied. In fact it is not difficult to check that these spaces $H^{2}(w)$ satisfy the hypotheses of Theorem 2.1, hence for them the conclusion of Theorem 3.4 is false. Note that if $\frac{1}{2}<a<1$, then a calculation with power series shows that $H^{2}(w)$ is one of the "heavily weighted Dirichlet spaces" of example (2) in $\S 1$.

3.6. PROOF OF THEOREM 3.4. We are assuming that $Y$ is an initial space and that the sequence $w$ satisfies (3.4.1) for some positive $\alpha<1$.

We first show that $Y_{w}$ satisfies the continuity axiom (X1). It follows from hypothesis (Y3) that each coefficient functional $\lambda_{n}$ is bounded on $Y_{w}$, with norm estimated by

$$
\left\|\lambda_{n}\right\| \leq M / w(n),
$$

where $M$ is a constant independent of $n$. Since $w$ is a positive, increasing sequence, it follows from (3.4.1) that $\sum w(n)^{-1}<\infty$, so by (3.6.1) we have for each $f \in Y_{w}$

$$
\sum|\hat{f}(n)| \leq\|f\|_{w} \sum\left\|\lambda_{n}\right\| \leq M \sum w(n)^{-1}<\infty .
$$

Thus the Taylor expansion of each $f$ in $X$ converges absolutely on the closed unit disc, so $Y_{w}$ satisfies $(\mathrm{X} 1)$.

To construct the desired composition operator, fix $\alpha<\mu<1$ and set

$$
\phi(z)=(1-\mu)+\mu z \quad(z \in U) .
$$

Thus $\phi$ maps the unit disc into itself, with $\|\phi\|_{\infty}=1$. We claim that $\phi$ induces a compact composition operator on $Y_{w}$.

For this purpose, a natural representation for $C_{\phi}$ on $H(U)$ is

$$
C_{\phi} f=\sum \lambda_{n}(f) \phi^{n} \quad(f \in H(U)),
$$

which exhibits $C_{\phi}$ as a limit of finite rank operators $T_{N}$ defined by

$$
T_{N} f=\sum_{1}^{N} \lambda_{n}(f) \phi^{n} \quad(f \in H(U)) .
$$


The $T_{N}$ 's operate on $Y_{w}$, since each power $\phi^{n}$, being a polynomial, belongs to $Y_{w}$. We will show that

$$
\sum\left\|\lambda_{n}\right\|\left\|\phi^{n}\right\|_{w}<\infty \quad\left(\left\|\lambda_{n}\right\|=\text { norm on } Y_{w}\right) .
$$

Granting this, it follows immediately that $T_{n} \rightarrow C_{\phi}$ in the operator norm of $Y_{w}$. Being the norm limit of finite rank operators, $C_{\phi}$ is therefore compact on $Y_{w}$ (in fact, by (3.6.3), it is actually nuclear $[14$, p. 476]).

To establish (3.6.3) it is enough, in view of (3.6.1), to prove that $\sum\left\|\phi^{n}\right\| / w(n)<$ $\infty$. To estimate the norm of $\phi^{n}$, apply successively the binomial theorem, the properties of norms, and hypothesis (Y4), which implies that $\sup \left\{\left\|z^{n}\right\|_{w} / w(n): n \geq\right.$ $0\}=M<\infty$. The result is

$$
\left\|\phi^{n}\right\|_{w} \leq M \sum_{k=0}^{n} C(n, k) \mu^{k}(1-\mu)^{n-k} w(k),
$$

where $C(n, k)$ is the appropriate binomial coefficient, and $M$ is independent of both $n$ and $\mu$. From this and the discussion above it follows that (3.6.3), and hence the compactness of $C_{\phi}$, will be established if we can prove

$$
\sum_{n=0}^{\infty} w(n)^{-1} \sum_{k=0}^{n} C(n, k) \mu^{k}(1-\mu)^{n-k} w(k)<\infty .
$$

To this end, let $\left(X_{j}: j \geq 1\right)$ be a sequence of independent random variables, each taking the value 1 with probability $\mu$, and 0 with probability $1-\mu$. Let $S_{n}=X_{1}+X_{2}+\cdots+X_{n}$, so that for each integer $0 \leq k \leq n$

$$
\operatorname{Prob}\left\{S_{n}=k\right\}=C(n, k) \mu^{k}(1-\mu)^{n-k} .
$$

It follows that the inner sum in (3.6.4) is just $\mathbf{E}\left\{w\left(S_{n}\right)\right\}$, the expectation of the random variable $w\left(S_{n}\right)$. So the target estimate (3.6.4) can be rewritten,

$$
\sum_{n=0}^{\infty} \mathbf{E}\left\{w\left(S_{n}\right)\right\} / w(n)<\infty
$$

The key to the proof of (3.6.5) is an inequality due to Hoeffding [10, p. 191], which states that if $\xi_{1}, \xi_{2}, \ldots, \xi_{n}$ are independent random variables with zero means and bounded ranges, say $a_{j} \leq \xi_{j} \leq b_{j}$, then for each $\lambda>0$

$$
\operatorname{Prob}\left\{\xi_{1}+\xi_{2}+\cdots+\xi_{n} \geq \lambda\right\} \leq \exp \left\{-2 \lambda^{2} / \sum_{j=1}^{n}\left(b_{j}-a_{j}\right)^{2}\right\} .
$$

Upon applying this inequality to the centered random variables $\xi_{j}=X_{j}-\mu$, we obtain after some rearranging

$$
\operatorname{Prob}\left\{S_{n} \geq \lambda\right\} \leq \exp \left\{-2(\lambda-n \mu)^{2} / n\right\} \text { for all } \lambda>n \mu .
$$

To employ this inequality, write

$$
\mathbf{E}\left\{w\left(S_{n}\right)\right\}=\sum_{k=0}^{n} w(k) \operatorname{Prob}\left\{S_{n}=k\right\} \equiv \sum_{1}+\sum_{2},
$$


where $\sum_{1}$ denotes the sum taken over $0 \leq k \leq[\alpha n]$, and $\sum_{2}$ the sum over the remaining $k$ 's. Since the sequence $w$ is increasing and the events $\left\{S_{n}=k\right\}$ are mutually exclusive, it is clear that $\sum_{1} \leq w([\alpha n])$.

In estimating the second sum it will be convenient to write $p(\lambda)$ for the left side of inequality (3.6.6), and $e(\lambda)$ for the right side. Note that $p(\lambda)=0$ for $\lambda>n$. Thus for $0 \leq k \leq n$

$$
\operatorname{Prob}\left\{S_{n}=k\right\}=p(k)-p(k+1) .
$$

We substitute this into the expression for $\sum_{2}$, sum by parts, use estimate (3.6.6) (which applies because $\alpha>\mu$, so the values $e(k)$ decrease for $k$ in the range of summation), and sum by parts again. The result is

$$
\begin{aligned}
\sum_{2} & \leq \sum^{\prime} w(k)(e(k)-e(k+1)) \quad\left(\sum^{\prime}=\text { sum over }[\alpha n]<k \leq n\right) \\
& \leq w(n) \sum^{\prime}(e(k)-e(k+1)) \quad(\text { since } w \text { is increasing) } \\
& =w(n)(e([\alpha n]+1)-e(n+1)) \\
& \leq w(n) e(\alpha n) \quad(\text { since } e(\lambda) \downarrow \text { for } \lambda>\mu n) \\
& =w(n) \exp \left\{-2(\alpha-\mu)^{2} n\right\} .
\end{aligned}
$$

These inequalities for $\sum_{1}$ and $\sum_{2}$ yield

$$
\mathbf{E}(w(S n))=\sum_{1}+\sum_{2} \leq w([\alpha n])+w(n) \exp \left(-2(\alpha-\mu)^{2} n\right),
$$

which, along with hypothesis (3.4.1) and the fact that $\alpha>\mu$, yields (3.6.5) and completes the proof of the theorem.

3.7. REMARKS. (a) The case $\alpha<\frac{1}{2}$ of Theorem 3.4 can be handled by setting $\mu=\frac{1}{2}$. In this case the sequence $\left(\xi_{j}\right)$ of random variables employed in the proof is a Rademacher sequence, and the corresponding special case of Hoeffding's inequality, is contained implicitly in Kahane's monograph [6, Chapter VI, §2].

(b) A slight modification of the argument given above shows that the function $\phi$ analyzed there induces a Hilbert-Schmidt composition operator on $H^{2}(w)$ whenever

$$
\sum_{n=0}^{\infty}(w([\alpha n]) / w(n))^{2}<\infty .
$$

The original proof, of course, shows that $C_{\phi}$ is in the trace class.

4. Final remarks. (a) Regarding Remark 2.4(b), the space $H^{2}(w)=H^{2}(R U)$ of Example 3.5(a) $\left(w(n)=R^{n}\right.$ for some $\left.R>1\right)$ has another interesting property: It supports a noncompact $C_{\phi}$ for which $\|\phi\|_{\infty}<1$ (cf. Remark $2.4(\mathrm{~b})$ ). In fact, the mapping $\phi(z)=z^{2} / R$ clearly induces such an operator. It would be of interest to know which of the spaces $Y_{w}$ support such operators.

(b) Theorem 2.1 and the main result of [7, Theorems 5.2 and 5.3] give an easy proof of the following necessary condition for the boundedness of composition operators on the heavily weighted Dirichlet spaces of example (2) in $\S 1$. If $\|\phi\|_{\infty}=1$ and $C_{\phi}$ is bounded on $D_{\alpha}$ for some $-1<\alpha<0$, then $\phi$ must have a finite angular derivative at some point of the unit circle. For example, $\phi$ cannot have norm 1 and map the unit disc into an inscribed polygon if it wishes to induce a bounded operator on one of these spaces. 


\section{REFERENCES}

1. L. Alpár, Egyes hatbánsyorok abszolút konvergenciája a konvergencia kör kerületen, Mat. Lapok 11 (1960), 312-322.

2. L. Carleson, Sets of uniqueness for functions regular in the unit circle, Acta Math. 87 (1952), 325-345.

3. J. A. Cima and W. Wogen, On algebras generated by composition operators, Canad. J. Math. 26 (1974), 1234-1241.

4. C. Cowen, Composition operators on $H^{2}$, J. Operator Theory 9 (1983), 77-106.

5. G. Halasz, On Taylor series absolutely convergent on the circumference of the circle of convergence. I, Publ. Inst. Math. Acad. Serbe Sci. 14 (1967), 63-68.

6. J. P. Kahane, Some random series of functions, Heath, Boston, Mass., 1968.

7. B. D. MacCluer and J. H. Shapiro, Angular derivatives and compact composition opertors on the Hardy and Bergman spaces, Canad. J. Math. 38 (1986), 878-906.

8. B. D. MacCluer, Composition operators on $S^{\rho}$, preprint.

9. E. A. Nordgren, P. Rosenthal, and R. S. Wintrobe, Composition operators and the invariant subspace problem, C. R. Math. Rep. Acad. Sci. Canada 6 (1984), 279-283.

10. D. Pollard, Convergence of stochastic processes, Springer-Verlag, New York, 1984.

11. R. C. Roan, Composition operators on the space of functions with $H^{p}$ derivative, Houston J. Math. 4 (1978), 423-438.

12. $\_$, Composition operators on a space of Lipschitz functions, Rocky Mountain J. Math. 10 (1980), 371-379.

13. H. J. Schwartz, Composition operators on $H^{p}$, Thesis, Univ. of Toledo, 1969.

14. J. H. Shapiro and P. D. Taylor, Compact, nuclear, and Hilbert-Schmidt composition operators on $H^{2}$, Indiana Univ. Math. J. 23 (1973), 471-496.

15. J. H. Shapiro, The essential norm of a composition operator, Ann. of Math. (to appear).

Department of Mathematics, Michigan State University, East Lansing, MICHIGAN 48824 